\title{
Risk factors for cardiovascular mortality and morbidity: The WHO multinational study of vascular disease in diabetes.
}

\author{
JH Fuller ${ }^{1}$, LK Stevens ${ }^{1}$, S-L Wang ${ }^{1}$ and the WHO Multinational Study Group* \\ ${ }^{1}$ EURODIAB, Department of Epidemiology and Public Health, University College, London, UK
}

\section{Abstract}

Aims/hypothesis. We aimed to examine the associations between classic cardiovascular risk factors and diabetes specific factors and the incidence of fatal and non-fatal end-points in a large cohort of diabetic patients.

Methods. A cohort of 4743 diabetic patients participating in the WHO Multinational Study of Vascular Disease in Diabetes (WHO MSVDD) has been followed up for about 12 years and the incidence of fatal and non-fatal cardiovascular disease outcomes assessed.

Results. The importance of blood pressure, serum cholesterol and proteinuria as predictors for cardiovascular disease mortality, fatal and non-fatal myocardial infarction and stroke is confirmed for patients with Type I (insulin-dependent) and Type II (non-insulin-dependent diabetes mellitus. Serum triglyceride was associated with cardiovascular disease death in Type II diabetes and in women with Type I diabetes and with MI incidence in Type II diabetes and stroke in Type II diabetic women. Fasting plasma glucose was associated with cardiovascular disease death, in- cidence of myocardial infarction and stroke in Type II diabetes only. In Type II diabetes, multivariate analysis confirmed that fasting plasma glucose was related to cardiovascular disease mortality independent of other risk factors. The independent relation of triglyceride to cardiovascular disease death was statistically significant only for Type II diabetic men. The presence of retinopathy was related to cardiovascular disease death and incidence of myocardial infarction in both types of diabetes and to stroke in Type II diabetes.

Conclusion/interpretation. This large cohort study shows that the assessment of cardiovascular disease risk in diabetes must include 'diabetes-related' variables such as glycaemic control, proteinuria and retinopathy, as well as the classic risk factors, blood pressure, smoking and dyslipidaemia. [Diabetologia (2001) 44 [Suppl 2]: S 54-S 64]

Keywords Type I (insulin-dependent) diabetes mellitus, Type II (non-insulin-dependent) diabetes mellitus, cardiovascular disease, stroke, blood pressure, smoking, cholesterol, triglyceride, proteinuria, myocardial ischaemia.
Corresponding author: Dr. J. Fuller, Department of Epidemiology and Public Health, University College London, 1-19 Torrington Place, London, WC1E 6BT, e-mail: harry@publicheath.ucl.ac.uk

Abbreviations: WHO MSVDD, Multinational Study of vascular Disease in Diabetes; CVD, Cardiovascular disease; MI, myocardial infarction; RR, realtive risk; MRFIT, Multiple Risk Factor Intervention Trial; WESDR, Wisconsin Epidemiologic Study of Diabetic Retinopathy; IHD, ischaemic heart disease.

* See reference 51 for list of investigators
Type I (insulin-dependent) and Type II (non-insulindependent) diabetes mellitus are characterised by an increased risk of cardiovascular disease (CVD) in men and women [1]. In particular there is an excess incidence of coronary heart disease (CHD) and stroke in people with diabetes. However the role of the cardiovascular factors increasing the risk of CVD in diabetes in the general population has remained controversial [2]. In order to plan cost-effective cardiovascular interventions in diabetic patients, the strength of the relation between various putative 
Table 1. Mean age and diabetes duration, and age-adjusted event rates (per 1000 person years) by Type I and Type II diabetes and by men and women

\begin{tabular}{llllll}
\hline & \multicolumn{2}{l}{ Type I (insulin-dependent) diabetes mellitus } & & \multicolumn{2}{c}{ Type II (non-insulin-dependent) diabetes mellitus } \\
\cline { 2 - 2 } & men $(n=659)$ & women $(n=601)$ & & men $(n=1661)$ & women $(n=1822)$ \\
\hline Mean (SD) & $44.4(6.1)$ & $44.5(6.2)$ & $47.0(5.7)$ & $46.7(5.6)$ \\
Age, years & $15.3(8.8)$ & $15.8(9.0)$ & $7.7(5.8)$ & $7.7(6.0)$ \\
Diabetes duration, years & $n^{\mathrm{a}}\left(\mathrm{Rate}^{\mathrm{b}}\right)$ & $n^{\mathrm{a}}\left(\mathrm{Rate}^{\mathrm{b}}\right)$ & & $n^{\mathrm{a}}\left(\mathrm{Rate}^{\mathrm{b}}\right)$ & $n^{\mathrm{a}}\left(\mathrm{Rate}^{\mathrm{b}}\right)$ \\
Events rates & $212(43.1)$ & $153(31.2)$ & & $408(25.5)$ & $326(17.8)$ \\
All-cause mortality & $95(20.4)$ & $62(12.6)$ & $210(13.3)$ & $156(8.4)$ \\
CVD mortality & $57(11.8)$ & $36(6.9)$ & $146(9.1)$ & $92(5.0)$ \\
Fatal MI & $64(15.6)$ & $49(11.8)$ & $206(16.0)$ & $159(10.4)$ \\
Fatal and non-fatal MI & $37(9.2)$ & $30(7.5)$ & $103(7.4)$ & $123(7.6)$ \\
Fatal and non-fatal stroke & & & &
\end{tabular}

a No. of events

${ }^{\mathrm{b}}$ events per 1000 person years

CVD risk factors and fatal and non-fatal end-points needs to be clarified [3]. The WHO Multinational Study of Vascular Disease in Diabetes (WHO MSVDD) is in a unique position to answer these questions in the context of a large international cohort of men and women with both types of diabetes mellitus.

\section{Subjects and methods}

Methods of patient selection and examination for the WHO MSVDD have been described in detail [4,5] and in this supplement [51]. The particular CVD risk factors included in this analysis were cigarette smoking, blood pressure, serum cholesterol and triglyceride, plasma glucose, proteinuria, retinopathy and ECG abnormalities. The methods of assessing these factors are described in detail elsewhere [51].

For our analysis retinopathy has been divided into two categories: any retinopathy or none.

For patients dying during the follow-up period, the underlying cause of death was coded according to the $9^{\text {th }}$ revision of the International Classification of Diseases (ICD-9). Cardiovascular deaths were those assigned to ICD 390-459 or 798.1, myocardial infarction (MI) deaths to ICD 410-414, and stroke deaths to ICD 430-438 [7]. Information on non-fatal MI and stroke was obtained from questionnaires completed at the reexamination phase [52].

Statistical analysis. To examine the association between the outcome variables (CVD mortality, fatal and non-fatal MI, and fatal and non-fatal stroke) and risk-factor categories, agestandardised event rates (per 1000 person years) were calculated, where the rates were directly standardised to the age distribution of the whole sample and event rates were compared between categories for all risk factors. Using the Cox proportional hazard model, age-adjusted relative risks (RRs) were also calculated for each category. For continuous variables the RR for a category was the risk of an event associated with having a value in that particular category relative to the risk of an event associated with having a value in the lowest category. For categorical variables the RR was the risk of an event associated with each category relative to the risk of an event when that risk factor was absent. The relative risk was calculated as the exponent of the beta coefficient of the risk factor in the
Cox proportional hazards model, where the model contained the risk factor as a categorical variable and age as a covariate. Any associations were considered statistically significant if the $95 \%$ confidence interval (CI) did not include a value of 1 $(p<0.05)$.

To render relative risks of continuous risk factors comparable, standardised estimates of relative risk (SRRs), adjusted for age, were also calculated. These were the relative risks associated with an increase in one standard deviation of the risk factor, again estimated by the Cox proportional hazard model. To assess the independent association of all risk factors with cardiovascular mortality, multivariate Cox proportional hazards modelling was carried out. Because serum triglyceride and plasma glucose were only measured in a sub-sample of 2097 and 3011 patients respectively, three multivariate models were done. Model 1 included age, duration of diabetes, systolic blood pressure (BP), serum cholesterol, smoking status, proteinuria, retinopathy and ECG abnormalities. In model 2 serum triglyceride was added to the variables in model 1 , and in model 3 plasma glucose was added instead of triglyceride. Serum triglyceride and plasma glucose were log transformed in these analyses because their distributions were skewed. Subgroups of patients had these latter two variables measured and the models which include these variables are restricted to these subgroups. The relative risks of these three models are comparable, however, as the basis of subgroup selection was geographic location, and not the relation between risk factor and outcome. Furthermore, a comparison of risk factor relation for shared variables between these three models indicates that these relative risks are similar and the populations included in the three models are therefore comparable. All analyses were carried out using the SAS statistical package.

\section{Results}

The mortality and morbidity follow-up was carried out in 4743 diabetic patients ( 2320 men and 2423 women), of whom 1260 (659 men and 601 women) were classified as having Type I diabetes and 3483 (1661 men and 1822 women) as having Type II diabetes. Before 1 January 1988, a total of 1099 (23\%) patients had died, $365(33 \%)$ of whom had Type I diabetes.

The mean age and duration of diabetes is given in Table 1 by sex and type of diabetes. This table also 
Table 2. Age-adjusted fatal and non-fatal MI and the relative risk (RR) associated with duration of diabetes, systolic blood pressure, serum cholesterol, serum triglyceride, plasma glucose, smoking, proteinuria, retinopathy and ECG abnormalities, by Type I and Type II diabetes and by men and women

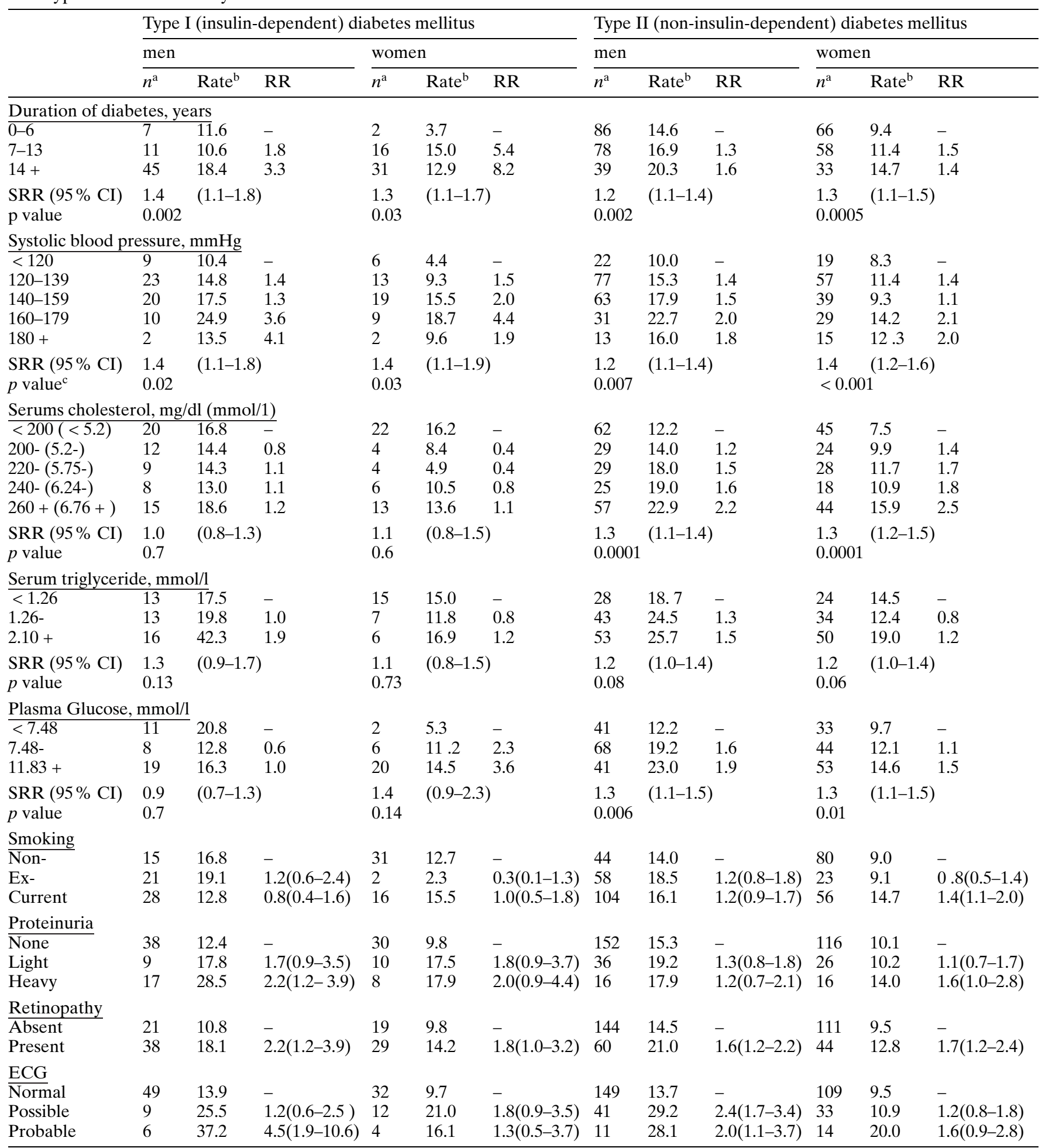

${ }^{\mathrm{a}}$ Number of events; ${ }^{\mathrm{b}}$ Age-adjusted rate per 1000 person years;

${ }^{c} p$-value relates to the significance of the risk factor as a continuous variable 
Table 3. Age-adjusted fatal and non-fatal stroke and the relative risk (RR) associated with duration of diabetes, systolic blood pressure, serum cholesterol, serum triglyceride, plasma glucose, smoking, proteinuria, retinopathy and ECG abnormalities, by Type I and Type II diabetes and by men and women

\begin{tabular}{|c|c|c|c|c|c|c|c|c|c|c|c|c|}
\hline & \multicolumn{6}{|c|}{ Type I (insulin-dependent) diabetes mellitus } & \multicolumn{6}{|c|}{ Type II (non-insulin-dependent) diabetes mellitus } \\
\hline & \multicolumn{3}{|l|}{ men } & \multicolumn{3}{|c|}{ women } & \multicolumn{3}{|l|}{$\overline{\text { men }}$} & \multicolumn{3}{|l|}{ women } \\
\hline & $\overline{n^{\mathrm{a}}}$ & Rate $^{\mathrm{b}}$ & RR & $n^{\mathrm{a}}$ & Rate $^{\mathrm{b}}$ & RR & $n^{\mathrm{a}}$ & Rate $^{b}$ & RR & $n^{\mathrm{a}}$ & Rate $^{\mathrm{b}}$ & RR \\
\hline \multicolumn{13}{|c|}{ Duration of diabetes, years } \\
\hline $0-6$ & 4 & 7.0 & _- & 2 & 2.7 & - & 50 & 7.9 & - & 49 & 6.6 & - \\
\hline $7-13$ & 11 & 11.5 & 2.3 & 6 & 6.8 & 2.3 & 33 & 6.7 & 0.9 & 46 & 8.2 & 1.4 \\
\hline $14+$ & 22 & 9.1 & 1.9 & 22 & 9.1 & 4.2 & 15 & 6.2 & 1.0 & 25 & 10.7 & 1.6 \\
\hline $\begin{array}{l}\text { SRR }(95 \% \mathrm{CI}) \\
p \text { value }\end{array}$ & $\begin{array}{l}1.2 \\
0.2\end{array}$ & \multicolumn{2}{|c|}{$(0.9-1.7)$} & $\begin{array}{l}1.4 \\
0.08\end{array}$ & \multicolumn{2}{|c|}{$(1.0-1.9)$} & $\begin{array}{l}1.0 \\
0.9\end{array}$ & \multicolumn{2}{|c|}{$(0.8-1.2)$} & $\begin{array}{l}1.2 \\
0.013\end{array}$ & \multicolumn{2}{|c|}{$(1.1-1.5)$} \\
\hline \multicolumn{13}{|c|}{ Systolic blood pressure, $\mathrm{mmHg}$} \\
\hline$<120$ & 5 & 8.6 & - & 3 & 3.9 & - & 9 & 3.7 & - & 20 & 8.7 & _- \\
\hline $120-139$ & 8 & 6.4 & 1.1 & 4 & 3.2 & 0.8 & 30 & 5.8 & 1.4 & 28 & 5.4 & 0.6 \\
\hline $140-159$ & 13 & 10.5 & 1.6 & 13 & 9.6 & 3.1 & 38 & 9.8 & 2.5 & 27 & 5.8 & 0.7 \\
\hline $160-179$ & 7 & 15.9 & 2.2 & 6 & 11.6 & 2.1 & 13 & 8.3 & 2.9 & 30 & 12.0 & 1.4 \\
\hline $180+$ & 4 & 22.1 & 4.5 & 4 & 22.0 & 8.1 & 13 & 15.7 & 4.6 & 18 & 14.8 & 1.5 \\
\hline SRR $(95 \%$ CI $)$ & 1.5 & \multirow{2}{*}{\multicolumn{2}{|c|}{$(1.1-2.0)$}} & \multirow{2}{*}{\multicolumn{3}{|c|}{$\begin{array}{l}1.8 \\
<0.001\end{array}(1.3-2.5)$}} & \multirow{2}{*}{\multicolumn{3}{|c|}{$\begin{array}{l}1.5 \\
<0.001\end{array}$}} & \multirow{2}{*}{\multicolumn{3}{|c|}{$\begin{array}{l}1.5 \\
<0.001\end{array}$}} \\
\hline$p$ value & 0.01 & & & & & & & & & & & \\
\hline \multicolumn{13}{|c|}{ Serum Cholesterol, $\mathrm{mg} / \mathrm{dl}(\mathrm{mmol} / \mathrm{l})$} \\
\hline$<200(<5.2)$ & 11 & 7.5 & - & 10 & 7.7 & - & 39 & 6.7 & - & 49 & 7.9 & - \\
\hline $200-(5.2-)$ & 11 & 15.7 & 1.8 & 3 & 5.2 & 0.7 & 12 & 5.5 & 0.8 & 22 & 8.6 & 1.1 \\
\hline $220-(5.75-)$ & 5 & 8.1 & 1.1 & 4 & 6.0 & 0.8 & 20 & 11.0 & 1.7 & 16 & 6.2 & 0.9 \\
\hline $240-(6.24-)$ & 5 & 9.8 & 1.3 & 2 & 3.6 & 0.5 & 11 & 7.3 & 1.1 & 10 & 5.6 & 0.8 \\
\hline $260+(6.76+)$ & 5 & 6.4 & 0.8 & 11 & 11.9 & 1.3 & 21 & 7.9 & 1.3 & 26 & 8.9 & 1.1 \\
\hline SRR $(95 \%$ CI) & 0.9 & $(0.7-1.3$ & & 1.3 & $(1.0-1.8$ & & 1.1 & $(0.9-1.4)$ & & 1.0 & $(0.8-1.2$ & \\
\hline$p$ value & 0.72 & & & 0.11 & & & 0.24 & & & 0.9 & & \\
\hline Serum Triglycer & $\mathrm{de}, \mathrm{mr}$ & $\mathrm{ol} / \mathrm{l}$ & & & & & & & & & & \\
\hline$<1.26$ & $\frac{46}{6}$ & 7.8 & - & 4 & 3.3 & - & 19 & 10.9 & - & 12 & 6.8 & - \\
\hline $1.26-$ & 5 & 7.9 & 1.0 & 3 & 4.8 & 1.5 & 14 & 6.9 & 0.6 & 26 & 8.3 & 1.3 \\
\hline $2.10+$ & 8 & 16.4 & 1.5 & 7 & 20.8 & 4.8 & 26 & 11.1 & 0.9 & 29 & 10.2 & 1.5 \\
\hline $\operatorname{SRR}(95 \% \mathrm{CI})$ & 0.9 & $(0.5-1.4$ & & 1.4 & $(0.8-2.4$ & & 1.1 & $(0.8-1.4)$ & & 1.2 & $(1.0-1.5$ & \\
\hline$p$ value & 0.53 & & & 0.19 & & & 0.50 & & & 0.07 & & \\
\hline Plasma Glucose & $\mathrm{mmol}$ & & & & & & & & & & & \\
\hline$<7.48$ & 4 & 9.3 & - & 2 & 6.6 & - & 20 & 5.6 & - & 18 & 4.9 & - \\
\hline $7.48-$ & 5 & 9.9 & 1.1 & 4 & 9.0 & 1.4 & 30 & 7.5 & 1.4 & 28 & 7.2 & 1.4 \\
\hline $11.83+$ & 13 & 10.1 & 1.8 & 6 & 3.9 & 0.9 & 26 & 13.8 & 2.5 & 42 & 11.1 & 2.3 \\
\hline $\operatorname{SRR}(95 \% \mathrm{CI})$ & 1.2 & $(0.8-1.9$ & & 0.9 & $(0.5-1.7$ & & 1.4 & $(1.1-1.8)$ & & 1.4 & (1.2-1.8 & \\
\hline$p$ value & 0.40 & & & 0.88 & & & 0.006 & & & 0.001 & & \\
\hline Smoking & & & & & & & & & & & & \\
\hline Non- & 5 & 6.3 & - & 16 & 7.4 & - & 14 & 4.2 & - & 64 & 6.6 & - \\
\hline Ex- & 8 & 7.3 & $1.0(0.3-3.2)$ & 5 & 8.8 & $1.3(0.5-3.7)$ & 30 & 8.3 & $2.1(1.1-4.0)$ & 24 & 9.0 & $1.2(0.8-2.0)$ \\
\hline Current & 24 & 11.6 & $1.9(0.8-5.1)$ & 9 & 6.9 & $1.1(0.5-2.5)$ & 59 & 8.5 & $2.2(1.2-3.9)$ & 35 & 8.9 & $1.3(0.9-2.0)$ \\
\hline Proteinuria & & & & & & & & & & & & \\
\hline None & 20 & 6.7 & - & 12 & 4.6 & - & 63 & 5.9 & - & 81 & 6.7 & - \\
\hline Light & 5 & 11.3 & $1.8(0.7-4.7)$ & 8 & 10.0 & $3.7(1$. & 18 & 9.1 & $1.6(1.0-2.8)$ & 26 & 9.5 & $1.6(1.1-2.5)$ \\
\hline Heavy & 11 & 18.1 & $2.9(1.4-6.1)$ & 9 & 22.0 & $5.2(2.0-13.2)$ & 19 & 18.8 & $3.8(2.3-6.4)$ & 15 & 12.8 & $2.4(1.4-4.2)$ \\
\hline Retinopathy & & & & & & & & & & & & \\
\hline Absent & 15 & 7.3 & - & 12 & 6.5 & _- & 66 & 6.2 & - & 78 & 6.2 & _- \\
\hline Present & 21 & 10.5 & $1.5(0.8-3.0)$ & 16 & 8.5 & $1.3(0.6-2.8)$ & 35 & 11.4 & $2.1(1.4-3.2)$ & 45 & 13.0 & $2.4(1.6-3.4)$ \\
\hline ECG & & & & & & & & & & & & \\
\hline$\overline{\text { Normal }}$ & 24 & 7.1 & - & 19 & 5.9 & - & 75 & 6.6 & - & 72 & 6.0 & - \\
\hline Pos & 8 & 20.0 & 2.2( & 7 & 11.3 & $1.7(0$ & 22 & 13.3 & $2.0(1$ & 41 & 13.2 & $2.1(1.4-3.1)$ \\
\hline Probable & 4 & 30.3 & $4.2(1.4-12.4)$ & 4 & 14.6 & $2.3(0.7-6.9)$ & 6 & 10.6 & $1.4(0.6-3.1)$ & 8 & 9.1 & $1.4(0.7-3.0)$ \\
\hline
\end{tabular}

${ }^{\mathrm{a}}$ Number of events; ${ }^{\mathrm{b}}$ Age-adjusted rate per 1000 person years;

${ }^{c} p$-value relates to the significance of the risk factor as a continuous variable 
Table 4. Multivariate analyses of relative risks for CVD mortality including age, duration of diabetes, systolic BP, serum cholesterol, smoking, proteinuria, retinopathy, and ECG abnormalities, by Type I and Type II diabetes and by men and women (Model 1)

\begin{tabular}{|c|c|c|c|c|}
\hline & \multicolumn{2}{|c|}{ Type I (insulin-dependent) diabetes mellitus } & \multicolumn{2}{|c|}{ Type II (non-insulin-dependent) diabetes mellitus } \\
\hline & $\operatorname{men}(n=605)$ & women $(n=521)$ & $\operatorname{men}(n=1502)$ & women $(n=1677)$ \\
\hline & $\mathrm{RR}(95 \% \mathrm{CI})$ & $\mathrm{RR}(95 \% \mathrm{CI})$ & $\mathrm{RR}(95 \% \mathrm{CI})$ & $\mathrm{RR}(95 \% \mathrm{CI})$ \\
\hline Systolic blood pressure & $1.4(1.1-1.8)$ & $1.5(1.1-2.0)$ & $1.2(1.1-1.4)$ & $1.3(1.1-1.5)$ \\
\hline Serum Cholesterol $^{\mathrm{a}}$ & $1.1(0.9-1.3)$ & $1.2(0.9-1.6)$ & $1.2(1.1-1.3)$ & $1.3(1.2-1.5)$ \\
\hline $\begin{array}{l}\text { Proteinuria } \\
\text { Light vs none } \\
\text { Heavy vs none }\end{array}$ & $\begin{array}{l}1.4(0.7-2.7) \\
2.0(1.1-3.5)\end{array}$ & $\begin{array}{l}1.6(0.7-3.5) \\
2.1(0.9-4.7)\end{array}$ & $\begin{array}{l}1.2(0.8-1.7) \\
1.6(1.1-2.5)\end{array}$ & $\begin{array}{l}1.6(1.1-2.4) \\
2.3(1.4-3.6)\end{array}$ \\
\hline $\begin{array}{l}\text { Retinopathy } \\
\text { Present vs absent }\end{array}$ & $1.1(0.7-1.9)$ & $1.3(0.7-2.5)$ & $1.4(1.1-2.0)$ & $2.3(1.6-3.3)$ \\
\hline
\end{tabular}

${ }^{\text {a }}$ Standardised relative risks

shows the numbers of events and age-adjusted event rates for all-cause mortality, CVD mortality, fatal MI, fatal and non-fatal MI, and fatal and non-fatal stroke, by sex and type of diabetes. Event rates in men or women with Type I diabetes were generally higher than in those with Type II diabetes. For both types of diabetes, rates were higher in men compared with women except for stroke incidence in Type II diabetes where the rates were similar.

Baseline risk factors and cardiovascular events - univariate analyses of fatal and non-fatal myocardial infarction. In patients of both sexes with Type II diabetes, duration of diabetes, systolic BP, cholesterol and plasma glucose significantly predicted the incidence of fatal and non-fatal MI (Table 2). For Type I diabetes, the significant predictors were duration and systolic BP in both sexes. Serum triglyceride was not significantly associated with this end-point in any subgroup, neither was smoking except for current smokers in Type II diabetic women. MI incidence was related to heavy proteinuria only in Type I diabetic men and to retinopathy in all sub-groups except women with Type I diabetes. Probable ischaemic ECG changes were predictive in men with Type I diabetes or Type II diabetes as were possible changes in Type II diabetic men.

Baseline risk factors and cardiovascular events - univariate analyses of fatal and non-fatal stroke. Systolic blood pressure was highly significantly associated with fatal and non-fatal stroke events for men and women with both types of diabetes (Table 3). Duration of diabetes was related to this end-point only in Type II diabetic women. Neither cholesterol nor tri- glyceride were related to stroke in patients with Type I diabetes or Type II diabetes. Plasma glucose was a predictor of stroke in men and women with Type II diabetes. Men with Type II diabetes who were current or ex-smokers had increased stroke rates as did all patients with heavy proteinuria and women with Type I diabetes or Type II diabetes with light proteinuria. Retinopathy was a risk factor for stroke in Type II diabetic men and women as were probable ECG changes in Type I diabetic men and possible changes in Type II diabetes for both sexes.

Baseline risk factors and cardiovascular mortality multivariate analysis. In Table 4 the relative risks are shown for CVD mortality estimated from the Cox proportional hazards model including age and all risk variables except serum triglyceride and plasma glucose (Model 1). The RRs for diabetes duration were only statistically significant for women with Type II diabetes. For systolic BP the relative risks (RRs) were greater than 1.0 for all sub-groups. Cholesterol was independently related to CVD death in men and women with Type II diabetes. For Type II diabetes only, ex-smoking men and current smoking men and women had increased RRs for CVD death. Heavy proteinuria was a significant independent contributor to CVD risk in Type I diabetic men and Type II diabetic men and women, as was light proteinuria in Type II diabetic women. The presence of retinopathy predicted CVD death in Type II diabetic men and women, independently of other risk variables. In this multivariate model, probable and possible ischaemic ECG changes were strongly predictive of CVD mortality in all sub-groups except in Type II diabetic women where probable changes were not significant. 


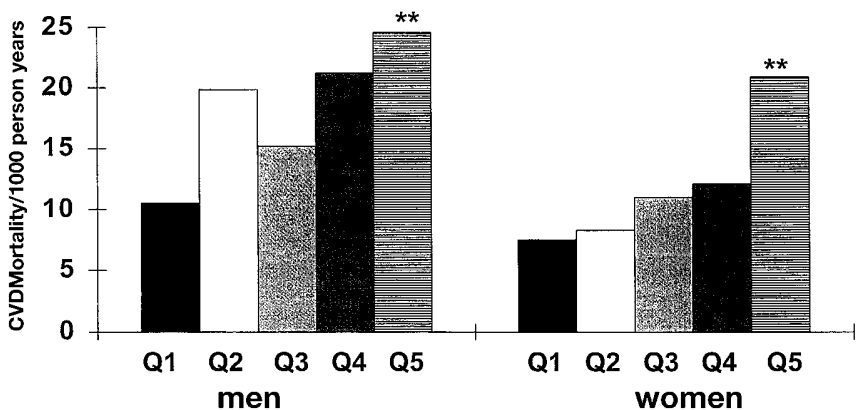

Fig. 1. Age-adjusted CVD mortality (per 1000 person years) for Type II diabetic men and women by quintile of fasting serum triglyceride $(\mathrm{mmol} / \mathrm{l}): \mathrm{Q} 1<1.10 ; \mathrm{Q} 2$ 1.11-1.50; Q3 1.51-2.02; Q4 2.03-2.93; Q5 > 2.94. Significantly different from $\mathrm{Q} 1 ;{ }^{* *} p<0.01$

The relation of CVD mortality to quintiles of fasting serum triglyceride is shown in Figure 1 for Type II diabetic patients. There was a positive association for both men ( $p$ for trend 0.001) and women ( $p$ for trend 0.0007). The results of the Cox proportional analysis for CVD mortality where serum triglyceride has been added to the model are shown in Table 5 (Model 2). The independent contribution of triglyceride to CVD risk was only significant for Type II diabetic men in this model. Systolic BP and retinopathy were independent predictors of CVD death only for Type I diabetic men and Type II diabetic women respectively. Duration, cholesterol and smoking status were not significant predictors in this model. Except for Type I diabetic women, heavy proteinuria remained an independent predictor as was light proteinuria in Type I diabetic men. Probable ECG changes (in Type I diabetes and Type II diabetic men) and

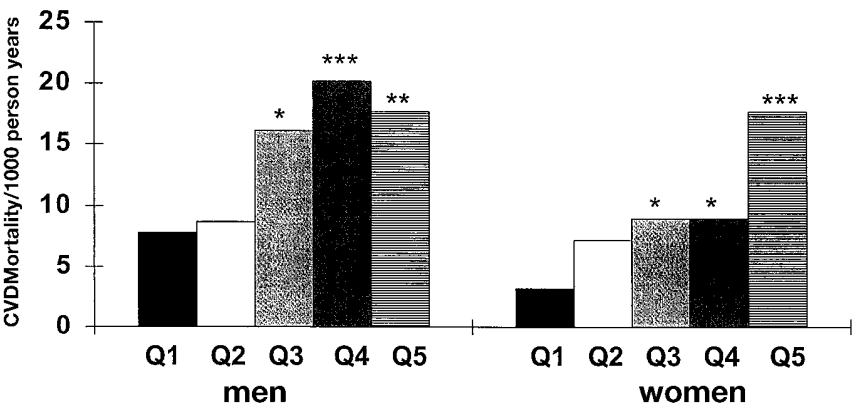

Fig. 2. Age-adjusted CVD mortality (per 1000 person years) for Type II diabetic men and women by quintiles of fasting plasma glucose $(\mathrm{mmol} /)$ : Q1 $<6.16 ; \quad$ Q2 6.17-7.92; Q3 7.93-9.90; Q4 10.00-13.09; Q5 > 13.10. Significantly different from Q1; ${ }^{*} p<0.05,{ }^{* *} p<0.01,{ }^{* * *} p<0.001$

possible changes (in Type II diabetic men and women) were also independent predictors of CVD death in this model.

Figure 2 shows the positive association between CVD mortality and quintiles of fasting plasma glucose for patients with Type II diabetes. The relation was significant for men ( $p$ for trend 0.0001) and women ( $p$ for trend 0.0001). In Table 6 plasma glucose replaces triglyceride in the multivariate analysis (Model 3) and it was independently related to CVD death in men and women with Type II diabetes, but not in Type I diabetes. Systolic BP was a significant independent predictor in all sub-groups except Type I diabetic men, as was cholesterol for Type II diabetic men and women. Duration of diabetes did not predict CVD death in this model and current smoking, heavy proteinuria and retinopathy were significant only for Type II diabetic women. Probable ECG changes in

Table 5. Multivariate analyses of relative risks for CVD mortality including all variables in Model 1 plus serum triglyceride (Model 2)

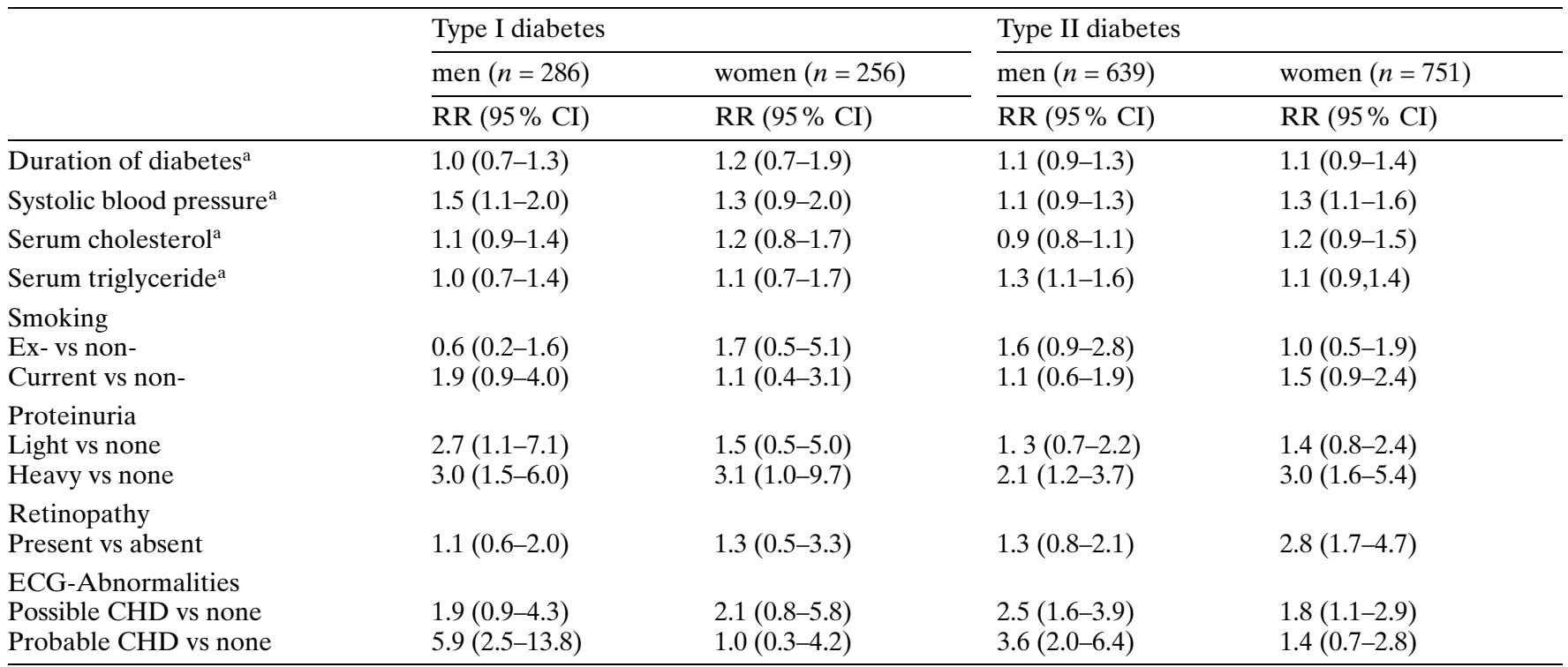

\footnotetext{
a Standardised relative risks
} 
Table 6. Multivariate analyses of relative risks for CVD mortality including all variables in Model 1 plus plasma glucose (Model 3)

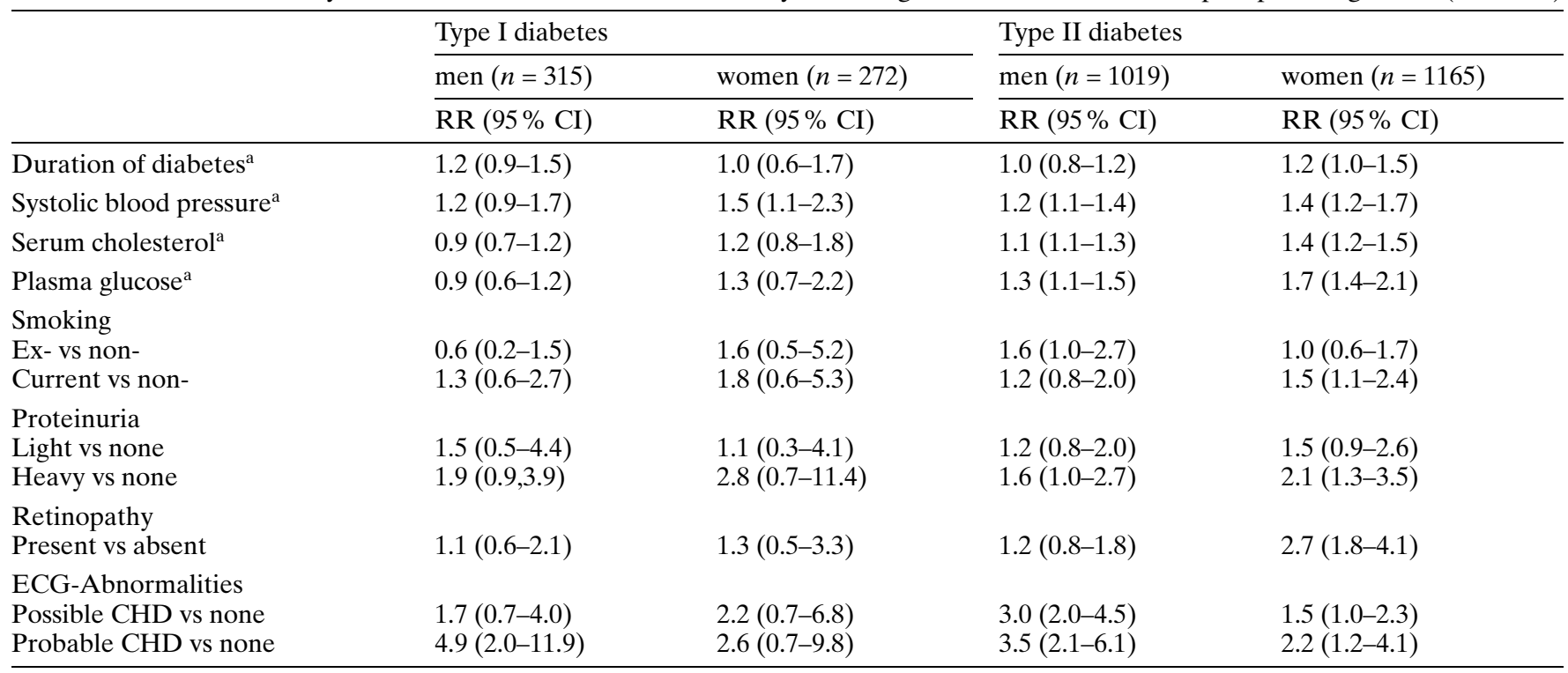

${ }^{a}$ Standardised relative risks

all sub-groups except women with Type I diabetes and possible changes in men with Type II diabetes were also predictors in this model.

Although both serum triglyceride and plasma glucose were correlated with body mass index (BMI), when the multivariate analyses were run with BMI included in Models 2 and 3 the associations of these variables to CVD risk were not altered.

In Figure 3 CVD mortality rates are plotted for patients with Type II diabetes according to the presence of one, two or three CVD risk factors comprising the following: Hypertension (systolic BP $\geq 140 \mathrm{mmHg}$ or diastolic $\mathrm{BP} \geq 90 \mathrm{mmHg}$ or on treatment); Hypercholesterolaemia (serum cholesterol $\geq 6.0 \mathrm{mmol} / \mathrm{l}$ ); current smoker.

The subjects are further sub-divided according to the presence or absence of proteinuria (heavy and light combined).

In those with no proteinuria, there was an increase in CVD risk according to the number of risk factors present in men ( $p$ for trend 0.0007) and women ( $p$ for trend 0.0002). The risk was further enhanced by the presence of proteinuria for all combinations of CVD risk factors.

\section{Discussion}

The increased cardiovascular mortality and morbidity associated with both types of diabetes, occurring particularly in developed countries, has been extensively documented $[1,8,9]$. In the last decade or so, at least twenty prospective studies have examined the strength of the associations between various putative risk factors and CVD mortality in diabetic popu- lations [10-28, 52, 53]. Comments have been made on the inconsistences in the findings of these studies even regarding the role of the classical CVD risk factors, blood pressure, serum cholesterol and cigarette smoking [2]. Of these only one small study was negative [25]; the largest one involving patients screened for the Multiple Risk Factor Intervention Trial (MRFIT) [14], the UK Prospective Diabetes Study (UKPDS) [52] and our study have clearly shown that all these three risk factors are predictive of CVD mortality in patients with Type II diabetes.

One factor contributing to these inconsistencies is clearly that of inadequate statistical power. Over one half of these studies have sample sizes less than 500 $[10,11,13,15-17,20,22,23,25,26,28,53]$, some much less, and only five have studied more than 900 patients $[12,14,19,24,52]$. In the large Wisconsin Epidemiologic Study of Diabetic Retinopathy (WESDR) [29] with 996 "younger onset" and 1370 "older onset" patients, the associations of ocular and systemic factors to total but not CVD mortality have been reported.

Another factor could be the variable characteristics of the studies in terms of diagnostic criteria, particularly for Type II diabetes, the methods of assessing risk factors and the availability of information on potentially important diabetes-related variables such as glycaemic control, urinary albumin or protein excretion, retinopathy and diabetes duration. This variability is particularly striking among the four largest studies. The Diabetes Intervention Study (DIS) did not use currently accepted diagnostic criteria for diabetes and the cohort (56\% men) was derived from patients selected for participation in a clinical trial [12]. All the MRFIT volunteers were men and the di- 

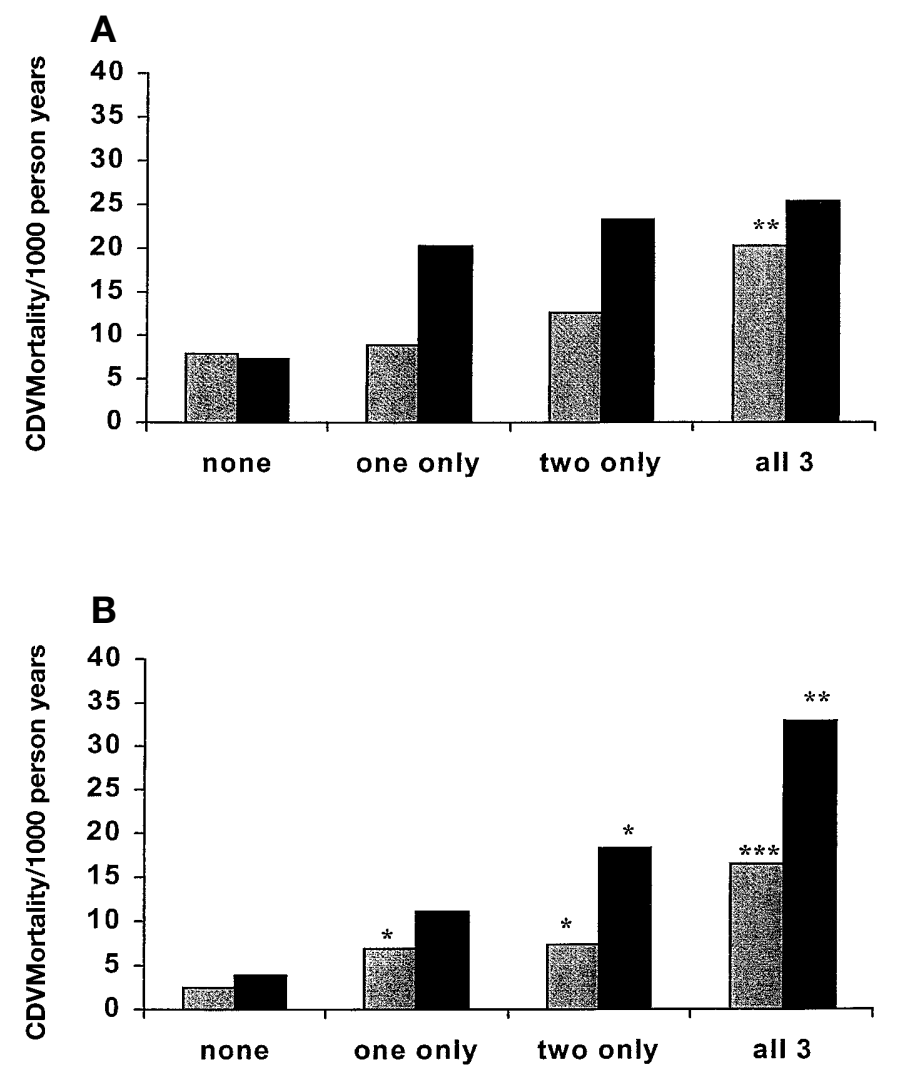

Fig.3 A, B. Age-adjusted CVD mortality (per 1000 person years) for (A) Type II diabetic men, (B) Type II diabetic women by presence of number of CVD risk factors (see text for definitions) and the presence of proteinuria. Significantly different from 'none'; ${ }^{*} p<0.05,{ }^{* *} p<0.01,{ }^{* * *} p<0.001$. negative, $\square$ positive

agnosis of diabetes was based on self-reported diabetes medication, thus excluding diet-treated subjects [14]. The diabetic women in the Nurses' Health Study were selected on the basis of a self-reported previous diagnosis of diabetes and risk factor status was assessed by a previous diagnosis of hypertension or high serum cholesterol [19]. The cohort of 943 men derived from the Paris Prospective Study consisted of 690 with impaired glucose tolerance (IGT), 158 with newly diagnosed diabetes and 135 with known diabetes [24]. The UKPDS cohort was recruited for participation in a clinical trial and of the original 7108 patients referred for study entry, 2006 (28\%) were excluded for various reasons including evidence of prevalent CVD, or late stage retinopathy or nephropathy [52]. In only one of these large studies [52] was the presence of retinopathy or raised urinary albumin or protein excretion assessed. Quality of glycaemic control was reported in only two of these studies $[12,52]$ and diabetes duration in only one [19]. The WHO MSVDD clearly compares well with other studies in terms of sample size, characterization of diabetic status and extent of risk factor and CVD end-point evaluation [4-7].
Evidence for the importance of raised blood pressure as a modifiable CVD risk factor in diabetes has been mounting over the years [30-32]. Of the five largest prospective studies discussed above, only the Paris study did not show that blood pressure was a predictor of CVD death [12, 14, 19, 24, 52]. Hypertension was associated with a relative risk of 1.82 for "diabetes-related deaths" in 3648 Type II diabetes participants in the Hypertension in Diabetes Study (HDS) [33] and to total mortality in WESDR [29]. Our study clearly shows that systolic BP has a strong and graded association with the risk of CVD death, fatal MI, and the incidence of MI and stroke in men and women with both types of diabetes. Multivariate analysis (Model 1) confirms the independent association of systolic BP with CVD death. Similar associations also hold for diastolic BP (data not shown).

Over recent years much evidence has accumulated in Type I and Type II diabetes linking proteinuria or raised urinary albumin excretion to an increased risk of cardiovascular as well as renal morbidity and mortality [34]. Of eight prospective studies which measured either proteinuria [21, 22, 26] or albuminuria $[10,11,16,17,53]$, only two $[16,53]$ did not find a significant association with CVD mortality. In the WHO MSVDD, proteinuria was detected by the semi-quantitative salicylsulphonic acid test which has a high sensitivity and specificity against urinary albumin measurements by radioimmunoassay [35]. Our study extends a previous analysis [36] by showing a strong association between heavy proteinuria and CVD mortality, fatal MI and stroke incidence in all study sub-groups and incidence of fatal and non-fatal MI in Type I diabetic men. Proteinuria is associated with increases of other risk factors, particularly blood pressure [36], but in the multivariate analysis its association with CVD death was independent of other risk factors, except in Type I diabetic women (Model 1). When compared with the general background population, WHO MSVDD participants with both hypertension and proteinuria had an extremely high mortality risk: 11-fold and 18-fold for Type I diabetic men and women respectively and 5-fold and 8-fold for Type II diabetic men and women respectively [37]. Even in the absence of hypertension and proteinuria, excess mortality was 2 -fold or more for patients with Type I diabetes or Type II diabetes. This finding does not support the contention that Type I diabetic patients with normal urinary albumin excretion might not be at increased risk of atherosclerotic morbidity [11].

Raised serum cholesterol is established with hypertension and smoking as being a major modifiable CVD risk factor in the general population [14]. However the situation has been uncertain for people with diabetes [2]. Out of seventeen prospective studies on Type II diabetes, only four larger ones $[14,19,24$, $52]$ and three smaller $[10,25,53]$ showed that serum 
total cholesterol was associated with CVD death. In our study cholesterol was a predictor of CVD mortality in Type I diabetes and Type II diabetes, and of MI incidence in Type II diabetes, but not of stroke. Information on the role of serum total triglyceride as a CVD risk factor in diabetes is available from ten prospective studies in Type II diabetes, seven showing statistically significant associations $[12,15,16,24,27$, $38,52]$ and three not $[13,17,53]$. In an earlier crosssectional analysis of baseline data from the WHO MSVDD, triglyceride was strongly related to Minnesota-coded ECG major Q-wave abnormalities [6]. In the present prospective study, triglyceride predicted CVD and fatal MI in men and women with Type II diabetes. The results were less consistent for Type I diabetes, but in the Pittsburgh cohort [38] triglyceride was associated with coronary events in univariate but not multivariate analysis. When triglyceride was entered into a multivariate analysis (Model 2), it remained an independent predictor of CVD mortality in Type II diabetic men, but not in the other subgroups. High-density-lipoprotein (HDL) cholesterol was not measured in the WHO MSVDD but was in seven other prospective studies in Type II diabetes, negatively related to CVD in two $[15,52]$ but not in the others $[10,13,16,17,53]$. The UKPDS has shown that low-density-lipoprotein (LDL) cholesterol is also an independent risk factor for coronary artery disease [52].

These inconsistences between studies on the role of total cholesterol and triglyceride in the cardiovascular risk associated with diabetes could be partly due to the previously mentioned problems of statistical power and study design. It could also be possible that the measurement of total lipids inadequately reflects the quantitative and qualitative abnormalities of lipoproteins occurring in Type I and Type II diabetes which could have important atherogenic potential $[1,40]$. In one of the few prospective studies of lipoprotein fractions and CHD risk in Type II diabetes it was shown that low HDL and $\mathrm{HDL}_{2}$ cholesterol, high very-low-density lipoprotein (VLDL) cholesterol, and high total and VLDL triglyceride were statistically significant risk factors [15]. They suggest that these atherogenic changes could precede the development of the chronic hyperglycaemia of Type II diabetes and are associated with insulin resistance [41].

For people with Type II diabetes, cigarette smoking was a statistically significant CVD risk factor in the WHO MSVDD, in four of the large prospective studies $[12,14,19,52]$ and in five of the smaller ones [16, 22, 25, 28, 53]. In Type I diabetes, smoking was not related to vascular disease in the Steno clinic cohort [11] nor in the present study. On the other hand in the Pittsburg study of Type I diabetes, heavy smoking was a risk factor for IHD death in women but not in men [23], and in WESDR smoking was associated with reduced survival in those with both "younger onset" and "older onset diabetes" [29].

Other researchers have reviewed the problems of distinguishing the role of hyperglycaemia as a cardiovascular risk factor in established diabetes [1]. A single assessment of glycaemic control might not reflect long-term exposure to hyperglycaemia, there could be a threshold effect and any direct effect of hyperglycaemia might be difficult to separate from those of associated factors such as abnormal lipoproteins, advanced glycation end-products (AGEs) or insulin resistance. Evidence from a number of prospective studies, using measures of fasting or post-prandial blood glucose or glycated haemoglobin, does suggest, however, that poor glycaemic control increases cardiovascular risk, particularly in Type II diabetes [10, $12,13,15-17,38,52,53]$. For the patients with Type II diabetes in our study, fasting plasma glucose was a predictor for all cardiovascular end-points, including stroke, and its association with CVD mortality was independent of all other variables (Model 3). A recent systematic review found that 23 out of 27 prospective studies in Type II diabetic patients showed a positive association between poor glycaemic control and either total or CVD mortality [54].

In the WHO MSVDD the presence of retinopathy was associated with several cardiovascular end-points in Type I and Type II diabetes and with stroke in Type II diabetes. This confirms similar findings in a Finnish study [42], the Pima Indians [21] and the Framingham population [43]. Several other prospective studies on Type II diabetes have shown that retinopathy is a predictor of all-cause mortality [29, 44-46]. There could be several explanations for this interesting association. Retinopathy could simply be associated with other CVD risk factors such as poor glycaemic control, hypertension, abnormal lipids or proteinuria. But the multivariate analysis confirmed that its association with CVD death was independent of these other factors. Alternatively the presence of retinopathy could be indicating the involvement of microvascular changes in the heart. But the role of small vessel disease in the aetiology of diabetic cardiomyopathy is not certain [46]. Finally retinopathy could be a marker of an underlying process, such as endothelial dysfunction, involved in the pathogenesis of both microvascular and macro-vascular disease. Support for this last hypothesis comes from the EURODIAB study on Type I diabetes where the presence or absence of retinopathy markedly affected the association between blood pressure and albumin excretion rate, itself thought to be a marker of endothelial dysfunction [48].

The contribution of duration of diabetes to CVD risk in Type II diabetes has been disputed in the past [49]. More recently seven prospective studies have examined the association of duration to cardiovascular risk in Type II diabetes and four showed a statisti- 
cally significant association $[13,15,19,21]$ and three did not $[17,22,27]$. In the WHO MSVDD, excess mortality increased with duration in Type II diabetes, allowing for the effect of age [37]. In our study the association in Type II diabetes between duration and CVD death was independent of age and other risk factors in women but not in men.

This analysis of 12-year morbidity and mortality data from the WHO MSVDD has confirmed the prognostic role of the classic risk factors, raised blood pressure, hypercholesterolaemia and smoking, in the increased CVD risk in people with diabetes. It also confirms the findings of the MRFIT study [14] that the presence of combinations of these classic CVD risk factors markedly increases CVD mortality in men and women with Type II diabetes, and shows that risk is enhanced still further by the presence of proteinuria. It has also shown that other "diabetes-related" factors besides proteinuria such as glycaemic control and retinopathy also contribute to risk status. Because of this more complicated risk profile in the diabetic patient, it would be ill-advised to apply one of the currently fashionable "risk tables" as a guide to preventative treatment since almost all of them are based on 25 year-old prognostic data from the Framingham study which regarded "diabetes" as a "yes/no" category [50]. The compilation of diabetesspecific CVD risk tables would be a much more valuable contribution to the prevention of this costly complication of diabetes [3].

\section{References}

1. Pyorala K, Laakso M, Uusitupa M (1987) Diabetes and Atherosclerosis: An Epidemiologic View. Diabetes Metab Rev 3: 463-524

2. Jarrett RJ (1992) Risk factors for coronary heart disease in diabetes mellitus. Diabetes 41 [suppl 2]: 1-3

3. Yudkin JS, Blauth C, Drury P et al. (1996) Prevention and Management of Cardiovascular Disease in Patients with Diabetes Mellitus: An Evidence Base. Diabetic Med 13: S101-S121

4. Jarrett RJ, Keen H, Grabauskas V (1979) The WHO Multinational Study of Vascular Disease in Diabetes: 1 General description. Diabetes Care 2: 175-186

5. Diabetes Drafting Group (1985) Prevalence of small vessel and large vessel disease in diabetic patients from 14 centres. The WHO Multinational Study of Vascular Disease in Diabetics. Diabetologia 28: 615-640

6. West KM, Ahuja MMS, Bennett PH et al. (1983) The role of circulating glucose and triglyceride concentrations and their interactions with other "risk factors" as determinants of arterial disease in nine diabetic population samples from the WHO Multinational Study. Diabetes Care 6: 361-369

7. Head J, Fuller JH (1990) International variations in mortality among diabetic patients: the WHO Multinational Study of Vascular Disease in Diabetes. Diabetologia 33: 477-481
8. Panzram G (1987) Mortality and survival in Type II (noninsulin-dependent) diabetes mellitus. Diabetologia 30: 123-131

9. Portuese E, Orchard TJ (1995) Mortality in Insulin-Dependent Diabetes. In: Harris MI et al. (ed) Diabetes in America. NIH, Washington, pp 221-232

10. Gall M-A, Borch-Johnsen K, Hougaard P, Nielsen S, Parving $\mathrm{H}-\mathrm{H}$ (1995) Albuminuria and poor glycaemic control predict mortality in Type II diabetes. Diabetes 44: 1303-1309

11. Deckert T, Yokoyama H, Mathiesen E et al. (1996) Cohort study of predictive value of urinary albumin excretion for atherosclerotic vascular disease in patients with insulin dependent diabetes. BMJ 312: 871-874

12. Hanefeld M, Fischer S, Julius U et al. (1996) Risk factors for myocardial infarction and death in newly detected Type II diabetes: the Diabetes Intervention Study, 11-year follow-up. Diabetologia 39: 1577-1583

13. Kuusisto J, Mykkanen L, Pyorala K, Laakso M (1994) Type II diabetes and its metabolic control predict coronary heart disease in elderly patients. Diabetes 43: 960-967

14. Stamler J, Wentworth D, Vaccaro O, Neaton JD (1993) Diabetes, other risk factors, and 12-yr cardiovascular mortality for men screened in the multiple risk factor intervention trial. Diabetes Care 16: 434-444

15. Laakso M, Lehto S, Penttila I, Pyorala K (1993) Lipids and Lipoproteins predicting coronary heart disease mortality and morbidity in patients with non-insulin-dependent diabetes. Circulation 88: 1421-1430

16. Uusitupa MIJ, Niskanen LK, Siitonen O, Voutilainen E, Pyorala K (1993) Ten-year cardiovascular mortality in relation to risk factors and abnormalities in lipoprotein composition in Type II (non-insulin-dependent) diabetic and non-diabetic patients. Diabetologia 36: 1175-1184

17. Standl E, Balletshofer B, Dahl B (1996) Predictors of 10year macrovascular and overall mortality in patients with Type II diabetes: the Munich General Practitioner Project. Diabetologia 39: 1540-1545

18. Ford ES, DeStefano F (1991) Risk factors for mortality from all causes and from coronary heart disease among persons with diabetes. Am J Epidemiol 133: $1220-1230$

19. Manson JE, Colditz GA, Stampfer MJ et al. (1991) A prospective study of maturity-onset diabetes mellitus and risk of coronary heart disease and stroke in women. Arch Intern Med 151: 1141-1147

20. Fitzgerald AP, Jarrett RJ (1991) Are conventional risk factors for mortality relevant in type 2 diabetes. Diabetic Med 8: 475-480

21. Nelson RG, Sievers ML, Knowler WC et al. (1990) Low incidence of fatal coronary heart disease in Pima Indians despite high prevalence of non-insulin-dependent diabetes. Circulation 81: 987-995

22. Morrish NJ, Stevens LK, Head J et al. (1990) A prospective study of mortality among middle-aged diabetics (the London cohort of the WHO Multinational Study of Vascular Disease in Diabetics) II: associated risk factors. Diabetologia 33: 542-548

23. Moy CS, LaPorte RE, Dorman JS et al. (1990) Insulin-dependent diabetes mellitus mortality. The risk of cigarette smoking. Circulation 82: 37-43

24. Fontbonne A, Eschwege E, Cambien F et al. (1989) Hypertriglyceridaemia as a risk factor of coronary heart disease mortality in patients with impaired glucose tolerance or diabetes. Diabetologia 32: 300-304

25. Adlerberth A, Rosengren A, Wilhelmsen L (1998) Diabetes and long-term risk of mortality from coronary and other 
causes in middle-aged Swedish men. Diabetes Care 21: 539-545

26. Krolewski AS, Kosinski EJ, Warram JH et al. (1987) Magnitude and determinants of coronary artery disease in juvenile-onset insulin-dependent diabetes. Am J Cardiol 59: $750-755$

27. Janka HU (1985) Five-year incidence of major macrovascular complications in diabetes mellitus. Horm Metab Res 15 [Suppl]: 15-19

28. Suarez L, Barrett-Connor E (1984) Interaction between cigarette smoking and diabetes mellitus in the prediction of death attributed to cardiovascular disease. Am J Epidemiol 120: 670-674

29. Klein R, Moss SE, Klein B, DeMets DL (1989) Relation of ocular and systemic factors to survival in diabetes. Arch Intern Med 149: 266-272

30. Fuller JH, Stevens LK (1991) Epidemiology of hypertension in diabetic patients and implications for treatment. Diabetes Care 14 [Suppl 4]: 8-12

31. Fuller JH, Stevens LK, Wang S-L (1996) Epidemiology of hypertension in diabetic patients. J Cardiovasc Pharmacol 28 [Suppl 4]: S1-S5

32. Curb JD, Pressel SL, Cutler JA et al. (1996) Effect of diuretic-based antihypertensive treatment on cardiovascular disease risk in older diabetic patients with isolated systolic hypertension. JAMA 276: 1886-1892

33. The Hypertension in Diabetes Study Group (1993) Hypertension in Diabetes Study (HDS): II. Increased risk of cardiovascular complications in hypertensive type 2 diabetic patients. J Hypertens 11: 319-325

34. Selby JV, Fitzsimmons SC, Newman JM, Katz PP, Sepe S, Showstack J (1990) The natural history and epidemiology of diabetic nephropathy. JAMA 263: 1954-1960

35. Watts GF, Hodgson B, Morris RW, Shaw KM, Polak A (1988) Side-room tests to screen for microalbuminuria in diabetes mellitus. Diabetic Med 5: 298-303

36. Stephenson JM, Kenny S, Stevens LK, Fuller JH, Lee E, WHO Multinational Study Group (1995) Proteinuria and mortality in diabetes: the WHO Multinational Study of Vascular Disease in Diabetes. Diabetic Med 12: 149-155

37. Wang S-L, Head J, Stevens L, Fuller JH, WHO Multinational Study Group (1996) Excess mortality and its relation to hypertension and proteinuria in diabetic patients: the WHO Multinational Study of Vascular Disease in Diabetes. Diabetes Care 19: 305-312

38. Lehto S, Ronnemaa T, Haffner S, Pyorala K, Kallio V, Laakso M (1997) Dyslipidemia and hyperglycaemia predict coronary heart disease events in middle-aged patients with Type II diabetes. Diabetes 46: 1354-1359

39. Lloyd CE, Kuller LH, Ellis D, Becker DJ, Wing RR, Orchard TJ (1996) Coronary artery disease in Type I diabetes. Gender differences in risk factors but not risk. Arterioscler Thromb Vasc Biol 16: 720-726

40. Taskinen M-R (1993) Quantitative and qualitative lipoprotein abnormalities in diabetes mellitus. Diabetes 41: 12-17

41. Reaven GM (1988) Role of insulin resistance in human disease. Diabetes 37: 1595-1607
42. Miettinen H, Haffner SM, Lehto S, Ronnemaa T, Pyorala K, Laakso M (1996) Retinopathy predicts coronary heart disease events in Type II diabetic patients. Diabetes Care 19: $1445-1448$

43. Hiller R, Sperduto RD, Podgor MJ, Ferris FL, Wilson PWF (1988) Diabetic retinopathy and cardiovascular disease in type II diabetics. Am J Epidemiol 128: 402-408

44. Knuiman MW, Welborn TA, Whittall DE (1992) An analysis of excess mortality rates for persons with non-insulin-dependent diabetes mellitus in Western Australia using the Cox proportional hazards regression model. Am J Epidemiol 135: 638-648

45. Hanis CL, Chu HH, Lawson K et al. (1993) Mortality of Mexican Americans with Type II diabetes. Retinopathy and other predictors in Starr County, Texas. Diabetes Care 16: 82-89

46. Neil A, Hawkins M, Potok M, Thorogood M, Cohen D, Mann J (1993) A prospective population-based study of microalbuminuria as a predictor of mortality in Type II diabetes. Diabetes Care 16: 996-1003

47. Bell DSH (1995) Diabetic Cardiomyopathy. A unique entity or a complication of coronary artery disease? Diabetes Care 18: 708-714

48. Stephenson JM, Fuller JH, Viberti G-C, Sjolie A-K, Navalesi R, The EURODIAB Type I diabetes Complications Study Group (1995) Blood pressure, retinopathy and urinary albumin excretion in Type I diabetes: the EURODIAB Type I diabetes complications study. Diabetologia 38: 599-603

49. Jarrett RJ (1984) Type II (non-insulin-dependent) diabetes mellitus and coronary heart disease - chicken, egg or neither? Diabetologia 26: 99-102

50. Haq IU, Jackson PR, Yeo WW, Ramsay LE (1995) Sheffield risk and treatment table for cholesterol lowering for primary prevention of coronary heart disease. Lancet 346 : 1467-1471

51. Lee ET, Keen H, Bennett PH, Fuller JH, Lu M, and the WHO Multinational Study Group (2001) Morbidity follow-up study of the WHO Multinational Study of Vascular Disease in Diabetes: general description and morbidity. Diabetologia 44 [Suppl 2]: S3-S13

52. Turner RC, Millns H, Neil HAW, Stratton IM, Manley SE, Matthews DR, Holman RR for the United Kingdom Prospective Diabetes Study Group (1998) Risk factors for coronary artery disease in non-insulin dependent diabetes mellitus: United Kingdom prospective diabetes study (UKPDS: 23). BMJ 316: 823-828

53. Florkowski CM, Scott RS, Moir CL, Graham PJ (1998) Lipid but not glycaemic parameters predict total mortality from Type 2 diabetes mellitus in Canterbury, New Zealand. Diabetic Med 15: 386-392

54. Groeneveld Y, Petri H, Hermans J, Springer MP (1999) Relation between blood glucose level and mortality in Type 2 diabetes mellitus: a systematic review. Diabetic Medicine 16: 2-13 